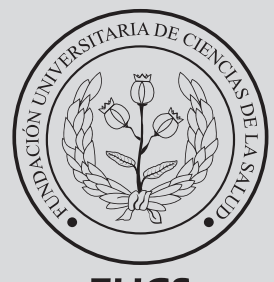

FUCS
Repr p

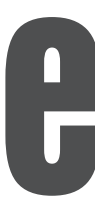

\title{
Conocimiento sobre la citohistotecnología en una institución prestadora de servicios de salud IV.
}

\begin{abstract}
Alex Alexi Flórez ${ }^{\mathrm{a}}$
Carlos Castro MD $^{\mathrm{b}}$

Rosa Stella Andrade ${ }^{c}$

Yuli Andrea Monroy ${ }^{\circ}$
\end{abstract}

\section{Knowledge on cytotechnology in a fourth level health care institution}

${ }^{a}$ Citohistología, Psicología, Especialista en Docencia Universitaria, Fundación Universitaria de Ciencias de la Salud. Bogotá DC, Colombia. ${ }^{b}$ Médico Epidemiólogo, Fundación Universitaria de Ciencias de la Salud. Bogotá DC, Colombia.

${ }^{c}$ Citohistología, Semillero de Investigación C\&T, Fundación Universitaria de Ciencias de la Salud. Bogotá DC, Colombia.

\section{R E S U M E N}

Introducción: el Ministerio de Educación Nacional (MEN) de Colombia reconoció 420 instituciones de educación superior (IES), de las cuales 243 (57.8\%) ofrecen programas técnicos o tecnológicos. El procesamiento de tejidos debe tener fundamento teórico y entrenamiento práctico para la preparación de tejidos humanos, animales o vegetales como apoyo diagnóstico. Esta profesión no es muy conocida y juega un papel relevante en el diagnóstico de patologías. Objetivo: determinar el conocimiento que tiene el personal de salud que labora en un hospital de cuarto nivel de atención con respecto a las habilidades y capacidades del citohistólogo. Métodos: estudio de corte transversal que incluyó trabajadores de una institución prestadora de servicios (IPS) de salud. Se calculó el tamaño muestral de 231 en Epi-info 7. Este trabajo fue aprobado por el comité de ética en investigación. Resultados: 231 trabajadores de la salud fueron interrogados, con una mediana de edad de 25 años (RIQ: 22-31), 83,1\% mujeres, 61,5\% conocen la definición correcta de citohistología y 49,3\% lo relacionan con un aporte en promoción y prevención de cáncer. Conclusiones: existe un desconocimiento por parte de los trabajadores de la IPS sobre las actividades del citohistólogo y sus funciones, como la adecuada fijación, traslado y procesamiento de los tejidos, que al no realizarse en forma correcta afectan el diagnóstico patológico, lo cual incide en la morbilidad por muestras inadecuadas, causando además trastornos administrativos hospitalarios.

Palabras clave: educación superior; profesión; Citohistología; conocimiento; citología; inmunohistoquímica; técnica histológica.

\section{INFORMACIÓN DEL ARTÍCULO}

Historia del artículo:

Fecha recibido: junio 19 de 2019

Fecha aceptado: noviembre 5 de 2019
Autor para correspondencia.

Sr. Alex Flórez

aaflorez@fucsalud.edu.co
DOI

10.31260/RepertMedCir.01217273.927 


\section{A B S T R ACT}

Introduction: the Colombian National Ministry of Education (MEN) recognized 420 higher education institutions (HEI) of which $243(57.8 \%)$ offer technical and technological study programs. Human or animal tissue preparation and processing as aids to diagnosis requires understanding its theoretical principles and practical training. This profession is little known and plays a relevant role for diagnosing diseases. Objective: to determine the knowledge among health care personnel of a fourth level institution regarding the skills and competencies of a cytotechnologist. Methods: a cross-section study including workers of a health care institution (IPS). Epi-info 7 was used to calculate the sample size of 231. This work was approved by the research ethics committee. Results: 231 health care providers answered a questionnaire, median age 25 years (IQR: 22-31), $83.1 \%$ females, of which, $61.5 \%$ know the correct definition of cytotechnology and $49.3 \%$ relate cytotechnology with cancer prevention and control [screening] programs. Conclusions: the health care personnel of the institution were not acquainted with the activities and functions of a cytotechnologist or the tissue fixation, processing and transportation procedures. An inappropriate sample can affect pathology results impacting morbidity rates causing hospital administrative issues.

Key words: higher education, profession; cytotechnology; knowledge; cytology; immunohistochemistry; histological technique.

(C) 2020 Fundación Universitaria de Ciencias de la Salud - FUCS. This is an open access article under the CC BY-NC-ND license (http://creativecommons.org/licenses/by-nc-nd/4.0/).

\section{INTRODUCCIÓ N}

La educación superior en Colombia comprende en el pregrado tres niveles de formación, la técnica profesional, tecnológica y profesional universitaria, y en el posgrado especialización, maestría y doctorado. Para el 2015 se reconocieron 420 instituciones de educación superior (IES), que se clasifican según su naturaleza de acuerdo con la ley 30 de 1992, en universidades 177 (42.1\%), instituciones universitarias $142(33.8 \%)$, instituciones tecnológicas $62(14.8 \%)$ y las técnicas profesionales $39(9.3 \%)$. En la misma línea, las carreras tecnológicas se relacionan con un conocimiento más específico, menos rutinarias y se caracterizan por desempeñar actividades en diferentes contextos con un grado mayor de complejidad. ${ }^{1-4}$

En el área de las ciencias de la salud, el procesamiento de tejidos provenientes de seres vivos hace necesario conocer el fundamento teórico y tener el entrenamiento práctico para la manipulación y preparación de tejidos humanos, animales o vegetales, que permiten su observación bajo el microscopio óptico como ayuda diagnóstica, constituyendo una herramienta de vital importancia para la detección de enfermedades dentro de las que se encuentran las patologías cancerígenas. ${ }^{5}$ La citología cervicovaginal es una prueba de tamizaje para exfoliar células del cuello uterino, enfocada a la prevención, diagnóstico y control de neoplasias cervicales que comprometen la salud de la mujer, en donde el citohistólogo es el responsable de la toma y el procesamiento de la muestra para que el patólogo bajo la visualización al microscopio pueda hacer un diagnóstico preciso..$^{6-8}$

Existen dificultades en la técnica del procesamiento histológico que se relacionan directamente con la manera de fijar los tejidos, las condiciones y los tiempos de traslados que son responsabilidad del personal de la salud ajeno al área de patología, lo que impacta en las características citomorfológicas y por consiguiente en la lectura por parte del patólogo. A pesar de esta importante labor y el frecuente contacto que tiene esta área de la salud con la población, no es claro el conocimiento y las actividades de esta profesión, por lo cual se este trabajo pretende describir el conocimiento del personal de salud que labora en el Hospital de San José con respecto a la profesión de la citohistología y sus funciones.

\section{MATERIALES Y MÉTODOS}

Se realizó un estudio de corte transversal, donde se incluyeron trabajadores (personal asistencial y administrativo) de una institución prestadora de servicios (IPS) de salud de IV nivel. Se excluyeron sujetos de las áreas de patología, servicios generales, seguridad y turno nocturno. Para determinar el tamaño de la muestra se efectuó un cálculo utilizando el software Epi-info 7, teniendo en cuenta los siguientes supuestos: población total 856 personas, prevalencia esperada de conocimiento sobre la carrera de citohistología $50 \%$, diferencia máxima esperada $5 \%$ y error tipo I $5 \%$, obteniendo una muestra de 221 trabajadores. Se adicionaron 10 sujetos más para un total de 231 con el fin de prevenir el sesgo de no respuesta. Después se realizó un muestreo aleatorio utilizando la base de datos referida por el departamento de recursos humanos de la IPS (total de colaboradores reportados por la firma MediCall, la cual no incluye la totalidad de los colaboradores del Hospital de San José). Se midieron variables demográficas y relacionadas con el conocimiento de la carrera de 
citohistología. La recolección de los datos fue realizada por estudiantes de los semilleros de investigación que fueron entrenados previamente. Las variables cualitativas se presentaron en frecuencias absolutas y relativas, y las cuantitativas en medidas de tendencia central y de dispersión, de acuerdo con la distribución de los datos. El análisis de la información se realizó en el software Stata 12. Este proyecto fue presentado y aprobado por el comité de ética en investigación con seres humanos.

\section{RESULTA D OS}

Se analizaron 231 trabajadores de una IPS de IV nivel, se encontraron 39 hombres (16,9\%) y 192 mujeres $(83,1 \%)$ entre 18 y 59 años y una mediana de 1 año (RIQ: 0.5 - 5) del tiempo que llevan laborando en la IPS. El resto de las características de la muestra se presentan en la tabla 1.

Tabla 1 . Características demográficas de la población a estudio

\begin{tabular}{|l|c|c|}
\hline \multicolumn{2}{|c|}{ (n=231) } \\
\hline Edad - med (RIQ)† años & $n$ & $\%$ \\
\hline Sexo & 25 & $(22-31)$ \\
\hline Femenino & 192 & 83,1 \\
Cargo & & \\
\hline Aprendiz/practicante $\ddagger$ & 7 & 3 \\
Auxiliar administrativo & 60 & 26 \\
Auxiliar de enfermería & 79 & 34,2 \\
Auxiliar de farmacia & 6 & 2,6 \\
Camillero & 5 & 2,2 \\
Enfermera jefe & 40 & 17,3 \\
Médico & 2 & 0,9 \\
Fisioterapeuta & 8 & 3,4 \\
Fonoaudiólogo & 1 & 0,4 \\
Instrumentación quirúrgica & 3 & 1,3 \\
Nutricionista & 2 & 0,9 \\
Secretaria & 13 & 5,6 \\
Auxiliar de morgue & 1 & 0,4 \\
Terapista respiratoria & 3 & 1,3 \\
Trabajadora social & 1 & 0,4 \\
Escolaridad & & \\
\hline Bachillerato & 8 & 3,5 \\
Técnico & 138 & 59,7 \\
Tecnólogo & 7 & 3 \\
Universitario & 70 & 30,3 \\
Especialización & 8 & 3,5 \\
\hline
\end{tabular}

† Mediana - Rango intercuartílico, $\ddagger$ Estudiante del Servicio Nacional de Aprendizaje (SENA).

Teniendo en cuenta que esta IPS de IV nivel hace parte de los campos de práctica de una institución de educación superior (IES) enfocada en ciencias de la salud, se preguntó acerca de los programas que ofrece la IES. De la población encuestada $(\mathrm{n}=231), 156(67,5 \%)$ refirió conocer alguno de los programas, de estos $(\mathrm{n}=156), 142(91 \%)$ conocen el programa de medicina, 139 (89.1\%) el programa de enfermería, 104 (45\%) el de instrumentación quirúrgica,
69 (29.9\%) el de psicología, 61 (24,4\%) el de citohistología, $48(20,8 \%)$ el de administración de empresas y $24(10,4 \%)$ el de atención prehospitalaria. De acuerdo con lo anterior $\mathrm{y}$ del total de los encuestados $(\mathrm{n}=231), 75$ colaboradores $(32,5 \%)$ mencionaron no conocer ningún programa.

Se preguntó: ¿Por qué medio de comunicación supo de los programas de la FUCS? Se encontró que $32(14 \%)$ se enteraron por internet, $3(1 \%)$ por televisión y el $81(85 \%)$ restante manifestó que otros medios de comunicación como voz a voz $81(63.7 \%)$, material publicitario $32(25,5 \%)$ y egresados $12(9,4 \%)$. 1 sujeto refirió ser estudiante de la IES.

En cuanto a la pregunta de: ¿Cuál es la definición de citohistología? Los resultados se presentan en la tabla 2.

Tabla 2 . Definición de citohistología

\begin{tabular}{|l|c|c|}
\hline \multicolumn{1}{|c|}{ Pregunta } & n & $\%$ \\
\hline $\begin{array}{l}\text { Estudio de las aplicaciones y los efectos de } \\
\text { las radiaciones }\end{array}$ & 4 & 1,7 \\
\hline $\begin{array}{l}\text { Estudio de células y preparación de tejidos } \\
\text { del cuerpo humano }\end{array}$ & 142 & 61,5 \\
\hline $\begin{array}{l}\text { Estudia las enfermedades que afectan al } \\
\text { ser humano }\end{array}$ & 18 & 7,8 \\
\hline $\begin{array}{l}\text { Es manipulación mecánica de las } \\
\text { estructuras anatómicas }\end{array}$ & 5 & 2,8 \\
\hline No sabe no responde & 62 & 26,8 \\
\hline
\end{tabular}

Dentro de las competencias que posee un citohistotecnólogo se encontró que $145(62,7 \%)$ lo relaciona con la capacidad para lectura de citologías y preparación de tejidos del cuerpo humano, $25(10,8 \%)$ con manejo de los equipos para la obtención de imágenes diagnósticas, 10 (4.3\%) lo ubican en un laboratorio clínico y el 51 (22,1\%) restante no respondieron la encuesta.

Con respecto a la pregunta: ¿Cree que existe alguna relación entre el estudio de cáncer de cuello uterino y la carrera de citohistología? De los encuestados 196 (84,8\%) respondieron que sí la existe y $35(15,1 \%)$ consideran que no. Frente a los objetivos de la profesión de citohistología la muestra encuestada refirió lo que se presenta en la figura 1.

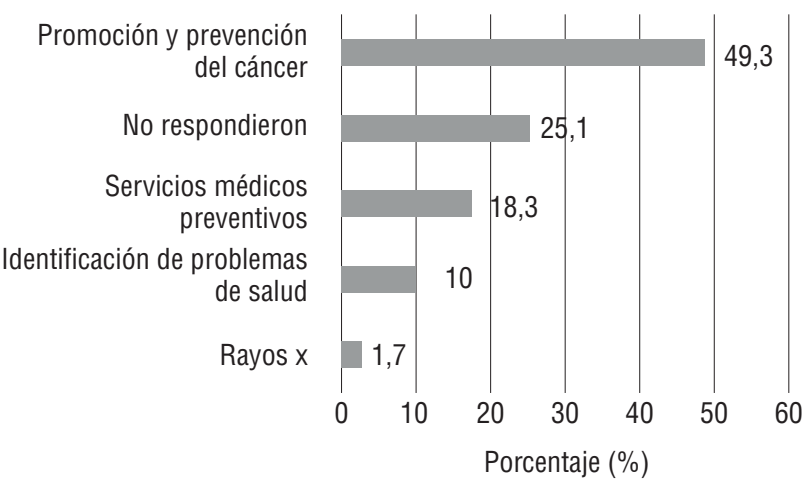

Figura 1. Conocimiento sobre los objetivos de la profesión de citohistología. 
En cuanto al número de semestres que componen la carrera de citohistología se observó que 206 (89.1\%) encuestados no tienen conocimiento, pues $3(1,3 \%)$ refieren dos semestres, $2(0,9 \%)$ tres semestres, $31(13,4 \%)$ cuatro semestres, 34 $(14,7 \%)$ seis semestres, $4(1,7 \%)$ siete semestres, $63(27,2 \%)$ ocho semestres, $3(1,3 \%)$ nueve semestres y $66(28,6 \%)$ diez semestres.

\section{DISCUSIÓN}

La finalidad de la educación técnica y tecnológica en el país según el MEN no consiste en formar sólo trabajadores sino ciudadanos con nuevas actitudes y capacidades, a través de currículos flexibles coherentes con las exigencias del entorno como condiciones para el avance de la competitividad. En Colombia la tasa de vinculación laboral para técnicos profesionales y tecnólogos según el "boletín de tendencia de las ocupaciones a nivel nacional y regional de 2015" fue del 55\%, que en comparación con el 2014 creció cerca de $10 \%$, evidenciando la competitividad de un técnico profesional frente a un profesional al momento de la vinculación con una empresa. Es importante mencionar que de la totalidad de vacantes según el sector productivo, la salud ocupa el $31 \%$. ${ }^{9,10}$

El incremento de las carreras técnicas y tecnológicas en los últimos años ha permitido que los jóvenes puedan acceder más fácil a estudios superiores teniendo en cuenta el costo y la duración, encontrando rápido oportunidades laborales con alta oferta en el mercado. Los ciclos propedéuticos dan la opción de trabajar paralelamente mientras avanzan en sus estudios, logrando en algunos casos la profesionalización. ${ }^{11.12}$

El procesamiento de tejidos y la lectura de citologías cervicovaginales (CCV) y de líquidos corporales son utilizados como herramientas de apoyo diagnóstico para la detección de enfermedades que pueden afectar la salud. Por ello adquiere gran importancia conocer quién es el responsable de estas funciones y la existencia de centros de formación universitaria que soportan la rigurosidad del quehacer del citohistólogo. ${ }^{13}$ Dentro de las funciones más reconocidas se encuentra la realización de $\mathrm{CCV}$ como prueba de tamizaje para la detección temprana de cáncer de cérvix, la cual es considerada como la segunda causa de muerte en mujeres a nivel mundial. ${ }^{14-16}$

En Colombia en la actualidad existen dos facultades que ofrecen el programa de citohistología: la Fundación Universitaria de Ciencias de la Salud con una trayectoria de 21 años y la Institución Universitaria Colegio Mayor de Antioquia. A nivel internacional la carrera es conocida en Venezuela por lo programas de las Universidades Arturo Michelena y Central de Venezuela, en Argentina la
Universidad CEMIT, en México la Universidad Autónoma de Sinaloa y Stony Brook en Nueva York, ${ }^{17}$ ofreciendo dos campos de acción: la citología y la técnica histológica.

A pesar de los esfuerzos realizados por la IES para darse a conocer, este estudio mostró que el programa de citohistología aunque se encuentra en el ámbito de la salud y hace parte de los programas ofrecidos es poco conocido, pues el $39.1 \%$ de los encuestados manifestó no tener conocimiento, lo cual se podría explicar por la falta de promoción de los programas en los colaboradores del hospital y por la trayectoria de carreras como medicina y enfermería, las cuales representan la mayor parte de estudiantes en la IES.

Finalmente, conocer en detalle esta profesión, no solo se convierte en un aspecto de cultura general, sino permite mejorar el manejo en el trasporte, procesamiento y diagnóstico, teniendo en cuenta que la citohistología como herramienta en el apoyo diagnóstico es fundamental para el tratamiento y pronóstico del paciente.

\section{Elementos relevantes para la reflexión}

- La educación técnica profesional y tecnológica actualmente son opciones competitivas al momento de seleccionar un programa de educación superior frente a las carreras profesionales.

- La citohistología es una profesión tecnológica que apoya el diagnóstico de patologías a partir del procesamiento de tejidos y lectura preliminar de citologías vaginales y líquidos corporales, así como el manejo de las nuevas técnicas de biología molecular, inmunofluorescencia e inmunostoquímica.

- Todos los profesionales de la salud, deben conocer esta profesión, pues juega un papel relevante en la cadena del diagnóstico, teniendo en cuenta que si se desconocen las condiciones de extracción, transporte y procesamiento, se puede afectar la validez diagnóstica.

- Este trabajo permitió fundamentar un nuevo proyecto para determinar el conocimiento sobre las condiciones de extracción, fijación y trasporte de los tejidos para evaluación patológica, esperando mejorar los procesos en esta área de la salud

- Las dificultades relacionadas con el procesamiento de tejidos no solo impacta las áreas administrativas de una IPS, sino afecta las características propias de la recolección de tejido por medio de biopsias, como riesgos quirúrgicos, anestésicos y eventos adversos relacionados directamente con el paciente.

\section{AGRADECIMIENTOS}

Agradecemos a los estudiantes Rosa Stella Andrade, Yuly Andrea Monroy, Jeisson Andrés Casallas Beltrán, Claudia Andrea Portilla, Yuly Natalia López Pérez, Jessica Lorena Llanos Duran, en la realización de este proyecto 


\section{CONFLICTO DE INTERESES}

Los autores declaran no tener ningún conflicto de intereses.

\section{REFERENCIAS}

1. Ministerio de Educación Nacional. Educación técnica y tecnológica para la competitividad Colombia. Colombia: Ministerio de Educación Nacional; 2008. p. 92.

2. Ministerio de Educación Nacional. Estadísticas de Educación Superior, Subdirección de Desarrollo Sectorial. In: Galvis B. CM, editor. Colombia: Ministerio de Educación Nacional; 2016. p. 4.

3. Ministerio de Educación Nacional. Instituciones de Educación Superior de Colombia [Internet]. Colombia: Ministerio de Educación Nacional; [cited 2019 junio]; Available from: https:// www.mineducacion.gov.co/portal/Educacion-superior/Sistemade-Educacion-Superior/231240:Instituciones-de-EducacionSuperior.

4. Ministerio de Educación Nacional. Niveles de la Educación Superior [Internet]. Colombia: Ministerio de Educación Nacional; [cited 2019 junio]; Available from: https://www.mineducacion. gov.co/portal/Educacion-superior/Sistema-de-EducacionSuperior/231240:Instituciones-de-Educacion-Superior.

5. Quintero L, Castro C. Perfil e inventario de personalidad de estudiantes de enfermería y su desempeño académico. Repert Med Cir. 2011;22(3):25-33.

6. Universidad de Antioquia. Definición y principio de método citológico [Internet]. Medellín: Universidad de Antioquia 1999 [cited 2019 junio]; Available from: http://docencia.udea.edu.co/ citologia/definicion.html.

7. Noticiero CM\& Internacional. Citología en base liquida [Video]. Video clip. Colombia: Noticiero CM\& Internacional, 2013.

8. Martín-Lacave I, García-Caballero T. Atlas de inmunohistoquímica: caracterización de células, tejidos y órganos normales. España: Ediciones Díaz de Santos; 2012.

\section{FINAN CIACIÓN}

Este proyecto fue financiado por una convocatoria interna de semilleros de investigación.
9. Servicio Nacional de Aprendizaje SENA. Tendencia de las Ocupaciones [Internet]. Colombia: Servicio Nacional de Aprendizaje SENA; 2015 [cited 2019 Junio]; Available from: https://observatorio.sena.edu.co/Tendencia/Boletines.

10. Servicio Nacional de Aprendizaje SENA. Observatorio. Colombia: Servicio Nacional de Aprendizaje SENA; 2015 [cited 2016 junio]; Available from: https://observatorio.sena.edu.co/.

11. Ministerio de Educación Nacional. Formación por ciclos propedéuticos [Internet]. Colombia: Ministerio de Educación Nacional; 2009 [cited 2016 junio]; Available from: https://www. mineducacion.gov.co/1621/article-196476.html.

12. elempleo. El impulso de las carreras técnicas y tecnológicas [Internet]. elempleo. 2011; Sect. Consejos profesionales

13. Guía global para la prevención y control del cáncer cervicouterino. Federación Internacional de Ginecología Oncológica (FIGO); 2009. p. 90.

14. Ministerio de Salud y Protección Social. Bajan tasas de incidencia y mortalidad de cáncer de cuello uterino en Colombia [Internet]. Colombia: Ministerio de Salud y Protección Social 2013 [cited 2016 junio]; Available from: https://www.minsalud.gov.co/Paginas/ Bajan-tasas-incidencia-mortalidad-cancer-cuello.aspx.

15. Ministerio de Salud y Protección Social, Instituto Nacional de Cancerología. Plan Decenal para el Control del Cáncer en Colombia, 2012-2021. Colombia: Ministerio de Salud y Protección Social; 2012. p. 124.

16. Fundación Universitaria de Ciencias de la Salud. Programa de Citohistología. Proyecto educativo. Bogotá: Fundación Universitaria de Ciencias de la Salud; 2011. 